\title{
BMJ Open Mutation screening of the TPO gene in a cohort of 192 Chinese patients with congenital hypothyroidism
}

Chunyun Fu, ${ }^{1,2}$ Bobo Xie, ${ }^{1,2}$ Shujie Zhang, ${ }^{1,2}$ Jin Wang, ${ }^{1,2}$ Shiyu Luo, ${ }^{1,2}$ Haiyang Zheng, ${ }^{1,2}$ Jiasun Su, ${ }^{1,2}$ Xuyun Hu, ${ }^{1,2}$ Rongyu Chen, ${ }^{1,2}$ Xin Fan, ${ }^{1,2}$ Jingsi Luo, ${ }^{1,2}$ Xuefan $\mathrm{Gu}^{3}{ }^{3}$ Shaoke Chen ${ }^{1,2}$

To cite: Fu C, Xie B, Zhang $\mathrm{S}$, et al. Mutation screening of the TPO gene in a cohort of 192 Chinese patients with congenital hypothyroidism. BMJ Open 2016;6:e010719.

doi:10.1136/bmjopen-2015010719

- Prepublication history for this paper is available online. To view these files please visit the journal online (http://dx.doi.org/10.1136/ bmjopen-2015-010719)

CF and BX contributed equally.

Received 1 December 2015 Revised 26 March 2016 Accepted 5 April 2016

CrossMark

For numbered affiliations see end of article.

Correspondence to Dr Shaoke Chen; chenshaoke123@163.com

\section{ABSTRACT}

Objectives: Defects in the human thyroid peroxidase (TPO) gene are reported to be one of the causes of congenital hypothyroidism $(\mathrm{CH})$ due to dyshormonogenesis. The aim of this study was to examine the TPO mutation spectrum and prevalence among patients with $\mathrm{CH}$ in the Guangxi Zhuang Autonomous Region of China and to define the relationships between TPO genotypes and clinical phenotypes.

Methods: Blood samples were collected from 192 patients with $\mathrm{CH}$ in the Guangxi Zhuang Autonomous Region, China and genomic DNA was extracted from peripheral blood leucocytes. All exons of the 10 common $\mathrm{CH}$-associated genes including TPO together with their exon-intron boundaries were screened by next-generation sequencing (NGS). The effect of the novel TPO mutation was investigated by 'in silico' studies.

Results: NGS analysis of TPO in 192 patients with $\mathrm{CH}$ revealed 3 different variations in 2 individuals (2/192, $1 \%)$. Sequencing other $\mathrm{CH}$ candidate genes in the patients with TPO variants revealed that patient 1 was homozygous for c.2422delT TPO mutation combined with double heterozygous DUOX2 pathogenic variants (p.R683L/p.L1343F) and patient 2 was triallelic for TPO pathogenic variants ( $\mathrm{p} . \mathrm{R} 648 \mathrm{Q} / \mathrm{p} . \mathrm{T} 561 \mathrm{M} / \mathrm{p}$. T561M). The present study identified a novel TPO variation c. $1682 \mathrm{C}>\mathrm{T} / \mathrm{p}$.T561M; and four known mutations: c.2422delT/p.C808Afs $\times 24$ and c.1943C $>$ T/ p.R648Q in TPO, c.2048G >T/p.R683L and c.4027C>T/ p.L1343F in DUOX2.

Conclusions: Our study indicated that the prevalence of TPO mutations was $1 \%$ among studied Chinese patients with $\mathrm{CH}$. More than two variations in one or more $\mathrm{CH}$-associated genes can be found in a single patient, and may, in combination, affect the phenotype of the individual. A novel TPO variation c.1682C $>T / p$. T561M was found, thereby expanding the mutational spectrum of the gene.

\section{INTRODUCTION}

Congenital hypothyroidism $(\mathrm{CH})$ is a common endocrine disorder with prevalence

\section{Strengths and limitations of this study}

- We conducted one of the largest TPO mutation screenings, in a cohort of 192 patients with congenital hypothyroidism $(\mathrm{CH})$, in the Guangxi Zhuang Autonomous Region, China.

- We identified that the prevalence of TPO pathogenic variants was $1 \%$ among patients with $\mathrm{CH}$.

- More than two variations in one or more $\mathrm{CH}$-associated gene can be found in a single patient, and the coexistence of multiple mutations may exacerbate the severity of the hypothyroid condition.

- We found a novel TPO variation, thereby expanding the mutational spectrum of the gene.

- The relationships between TPO genotypes and clinical phenotypes could not be clearly determined because only two patients were identified to be mutation-positive participants and both had multiple variations.

ranging from 1:2000 to 1:4000 newborns. ${ }^{1}$ Apart from iodine deficiency, which is still a major cause for endemic hypothyroidism in neonates, ${ }^{2}$ the sporadic $\mathrm{CH}$ cases can be classified into two groups: (1) disorders of thyroid gland development (thyroid dysgenesis) that have been linked to mutations in TSHR, PAX8, NKX2.1 and FOXE1 genes, ${ }^{3}$ which account for the majority of cases (80$85 \%)^{3}{ }^{4}$; and (2) abnormalities in thyroid hormone synthesis (dyshormonogenesis), which account for the remaining 15-20\% of cases, ${ }^{5}$ and are associated with mutations in DUOX2, TG, TPO, SLC5A5, SLC26A4 and IYD genes. $^{6}$

Defects in the human TPO (NM_000547) gene are reported to comprise some of the most common causes of thyroid dyshormonogenesis (TDH) ${ }^{7-10}$ TPO, a thyroid-specific haeme peroxidase localised in the apical membrane of thyrocytes, plays a vital role in thyroid hormone biosynthesis. $\mathrm{CH}$ caused by mutations in the TPO is an autosomal 
recessive disorder, and most patients with biallelic TPO mutations have permanent congenital hypothyroidism (PCH). Up to now, about 100 mutations have been reported and recorded in the HGMD (http://www. hgmd.cf.ac.uk/ac/index.php), but the genotype-phenotype relationship has not yet been fully established, and little is known about its mutational spectrum and prevalence among Chinese patients with $\mathrm{CH}$. We performed the TPO gene screening in a cohort of 192 patients with $\mathrm{CH}$ in Guangxi Zhuang Autonomous Region, China.

\section{MATERIALS AND METHODS}

\section{Patients}

We enrolled 192 patients (101 females and 91 males) with $\mathrm{CH}$; the previous 45 patients with $\mathrm{CH}$ collected for DUOX2 mutation screening were not included in this study. ${ }^{11}$ Most patients were initially identified by neonate screening among 623000 newborns in the Guangxi Zhuang Autonomous Region, China, from October 2009 to June 2014. The thyroid gland was normal in size and position in 73 of these patients; 72 patients had increased and 47 showed decreased size of the thyroid gland. Newborn screening was performed with filter paper for $\mathrm{CH}$ between $72 \mathrm{~h}$ and 7 days after birth. Blood samples were collected from the heel and the thyroidstimulating hormone (TSH) level was measured by timeresolved fluorescence assay (Perkin Elmer, USA). Newborns with increased TSH (TSH $\geq 8 \mathrm{mIU} / \mathrm{L}$ ) levels observed during neonatal screening were followed-up for further evaluation. Serum TSH and FT4 were determined by electrochemiluminescence assay (Cobas e601, Roche Diagnostics, USA). Diagnosis of $\mathrm{CH}$ was based on elevated TSH levels (TSH $>10 \mathrm{mIU} / \mathrm{L})$ and decreased FT4 levels (FT4 $<12 \mathrm{pmol} / \mathrm{L})$. Permanent or transient $\mathrm{CH}$ was determined using results of thyroid function tests after temporary withdrawal of L-T4 therapy at 2 years of age. The perchlorate discharge test, which aids in the recognition of iodide organification defects, is not routinely performed. The study was approved by the Medical Ethics Committee of Guangxi Maternal and Child Health Hospital. Informed consent was obtained from the parents of the patients.

\section{Mutation detection and interpretation}

Peripheral venous blood samples were collected from the patients. Genomic DNA was extracted from peripheral blood leucocytes, using QIAamp DNA Blood Mini Kit (Qiagen, Germany) according to the manufacturer's protocol. A $\mathrm{CH}$ capture panel an Illumina Truseq Custom Amplicon V.1.5 kit, included 10 known CH-associated genes (TPO, TSHR, PAX8, NKX2.1, FOXE1, DUOX2, TG, SLC5A5, SLC26A4 and IYD) with complete coding regions and flanking intronic regions; the DUOXA2 gene was not included. The prepared sample library was sequenced employing an Illumina MiSeq instrument using MiSeq Reagent Kit V.2, 500-cycles (Illumina Inc, San Diego, California, USA).
Illumina Amplicon Viewer V.1.3 and MiSeq Reporter V.2.3 software were used for data analysis, and the SnpEff ${ }^{12}$ was used for variant annotation. Polyphen $2^{13}$ and MutationTaster ${ }^{14}$ were used to evaluate the pathogenicity of the novel mutations. DNAMAN software V.8 was then used to carry out multiple sequence alignment of the TPO family protein from different species. In addition, a cohort of 300 ethnicity-matched healthy participants was used to assess the variant frequencies in the normal control. All control participants had normal FT4 and TSH levels.

\section{Sanger sequencing}

Sanger sequencing was used to validate the mutations identified from next-generation sequencing.

\section{In silico studies of the novel TPO gene mutation}

The crystal structure of human TPO is currently not available. To study the three-dimensional (3D) structure of the p.T561M TPO, we modelled the wild-type and mutant TPO variants in silico, using the Swiss Model program package. ${ }^{15} 16$ Multiple templates were used to generate different target models, and the one presenting the best sequence identity percentage was chosen. Thus, a homology model was generated with human myeloperoxidase isoform $\mathrm{C}$ (PDB accession number $1 \mathrm{mhl}$ ) as a template. Myeloperoxidase showed an overall identity of $48.16 \%$ for amino acids 258-735 of TPO. Verify3D software was used to determine the compatibility of the 3D atomic model with its own 1D amino acid sequence. The structure with lowest potential energy was extracted, verified using Ramachandran plots that were generated by Procheck software (http://nihserver.mbi. ucla.edu/SAVES), to check the stereo chemical quality of the protein. We then submitted the chosen model to the Swiss PDB Viewer ${ }^{17}$ to determine potential structural differences between the mutant and the wildtype TPO.

\section{RESULTS}

\section{Analysis of TPO and other $\mathrm{CH}$-associated genes by} next-generation sequencing

PCR-based targeted enrichment, using Illumina Truseq Custom Amplicon, of 10 known $\mathrm{CH}$ genes followed by 500 cycles paired-end sequencing on an Illumina MiSeq was performed, resulting in a total of 4.77 million reads. The average coverage is more than 95\%; all TPO exon depths were successfully covered with $>40 \times$. Also, no deletion or duplication of exons in TPO was detected by manual read depth inspection. In this study, we identified three different TPO variants in two individuals. Sequencing of other $\mathrm{CH}$ candidate genes in the two patients with the TPO mutations showed that patient 1 was homozygous for c.2422delT TPO mutation combined with double heterozygous DUOX2 mutations (p.R683L/ p.L1343F), and patient 2 was triallelic with TPO pathogenic variants (p.R648Q/p.T561M/p.T561M). All variants were confirmed by Sanger sequencing (figures 1 
Figure 1 Pedigree of the affected family 1 and Sanger sequencing for validation of the mutations detected by the next-generation sequencing platforms.
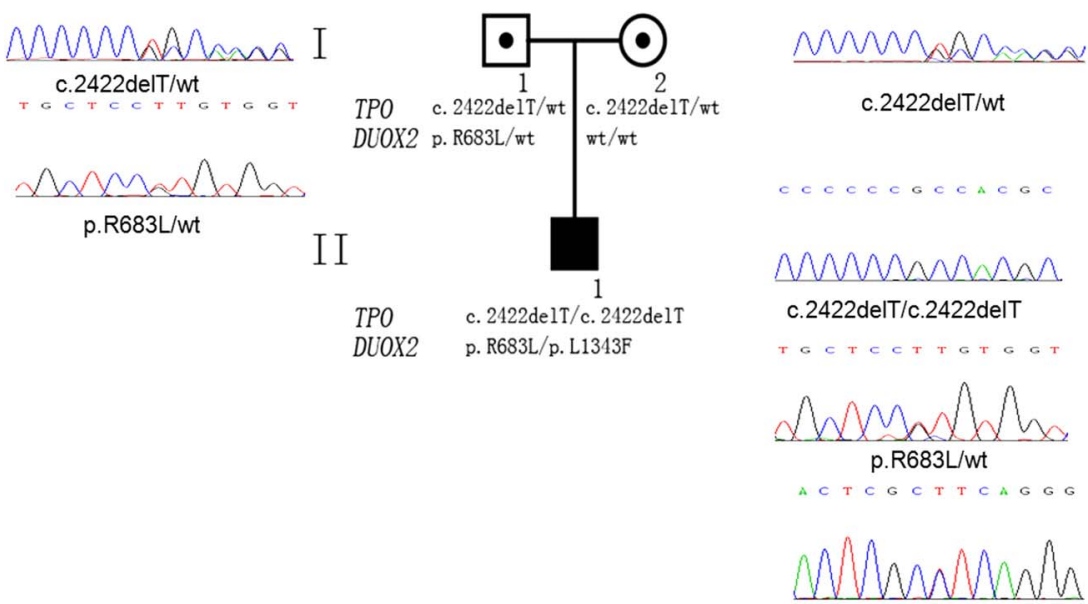

p.L1343F/wt and 2). The present study identified a novel variation: $p$. T561M in the TPO; and four known mutations: c.2422delT and p.R648Q in TPO, p.R683L and p.L1343F in DUOX2. Polyphen2 and Mutation taster predicted that the novel variation p.T561M would have deleterious effects by damaging the TPO protein. Additionally, the variant was not detected in our normal control population. DNAMAN software was then used to carry out multiple sequence alignment of the TPO family protein from different species; the identified variation was found to be located in the highly conserved region of TPO (figure 3). These all suggested that the amino acid substitution might be due to a pathological mutation.

\section{In Silico predictions of functional impact of the T561M on the TPO protein}

The generated 3D homology models based on $1 \mathrm{mhl}$ template ( $44 \%$ sequence similarity) covered $50 \%$ of the full sequence of TPO protein. Verify3D showed that the model had passed as a good model by having $86.6 \%$ of residues with an average $3 \mathrm{D}$ to $1 \mathrm{D}$ score of more than 0.2. Ramachandran plots from Procheck showed that the model had three amino acid (Gly331, Glu384 and
Lys627) residues located in the disallowed regions. However, these amino acid residues were distant from the active site. Consequently, the homology model was acceptable for subsequent analyses.

The threonine 561 residue is located within a highly conserved region of TPO, which suggests an important role in the function and/or structure of TPO. Comparison between predicted tertiary structures of the wild type and mutant proteins revealed that the substitution, p.T561M, breaks two hydrogen bonds with an asparagine in position 557 and another hydrogen bond with a glutamic acid in position 558. Significant structural alterations in the annotation were also detected in the p.Thr561Met mutant (figure 4). As a result, these changes will disturb the secondary structure and affect the function of TPO protein.

\section{Clinical features and laboratory test results of the patients}

The clinical features and laboratory results are summarised in table 1. Patient 1 was born at full-term to non-consanguineous parents. His birth weight was $3000 \mathrm{~g}$ and length $47 \mathrm{~cm}$. There was no family history of thyroid disease. Newborn screening at the age of 6 days
Figure 2 Pedigree of the affected family 2 and Sanger sequencing for validation of the mutations detected by the next-generation sequencing platforms. NA, data are not available.

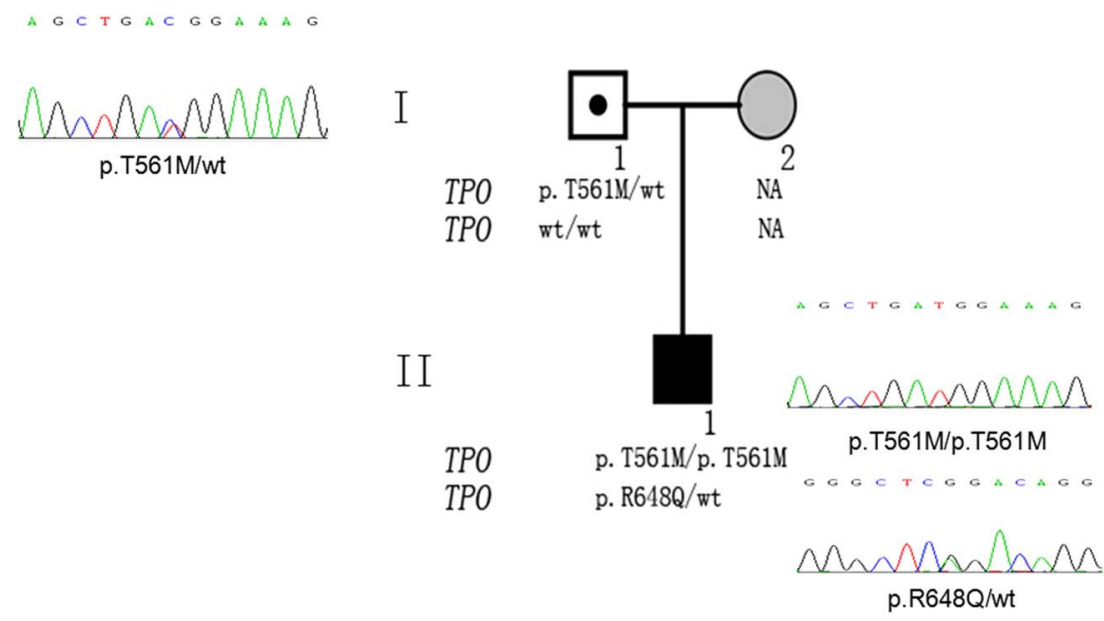


Figure 3 Multiple sequence alignment of TPO from different species. The arrow indicates that the threonine 561 residue is located within a highly conserved region.

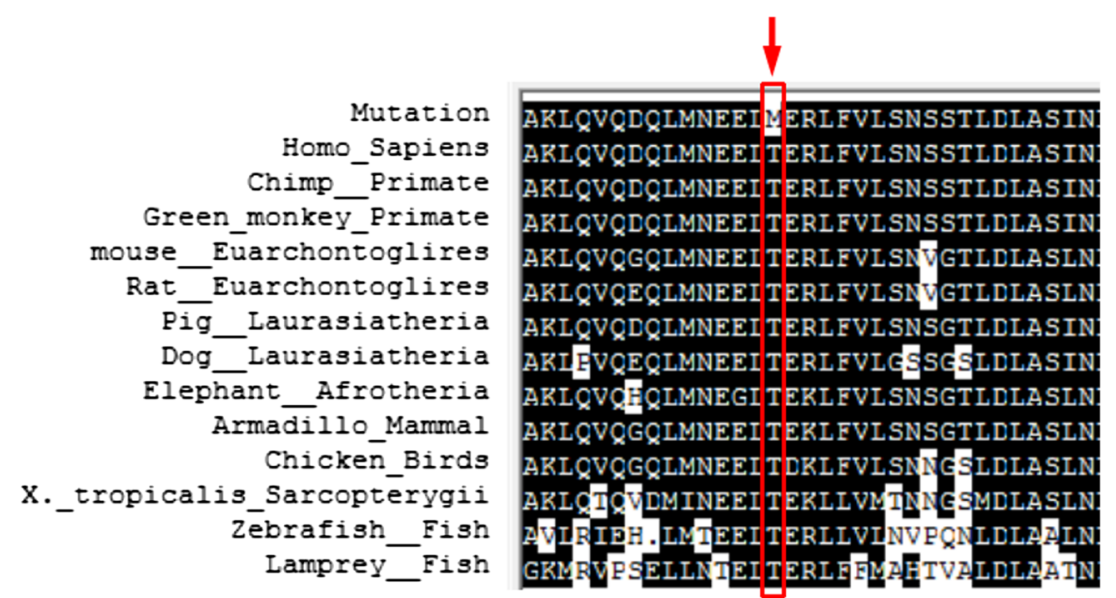

revealed a high level of $\mathrm{TSH}(78.6 \mathrm{mIU} / \mathrm{L})$. He was therefore re-evaluated at hospital at the age of 13 days. A serum TSH level of $>100 \mathrm{mlU} / \mathrm{L}$ (normal range $0.7-$ $10 \mathrm{mIU} / \mathrm{L}$ ), FT4 level of $1.92 \mathrm{pmol} / \mathrm{L}$ (normal range 12-32 pmol/L) and FT3 level of $1.68 \mathrm{pmol} / \mathrm{L}$ (normal range $2.65-9.68 \mathrm{pmol} / \mathrm{L})$ confirmed the diagnosis. Ultrasound examination showed an enlarged thyroid gland (right lobe $3.4 \times 1.5 \times 1.4 \mathrm{~cm}$; left lobe $3.2 \times 1.4 \times 1.3 \mathrm{~cm})$. L-T4 replacement therapy was started immediately at an initial daily dose of $12 \mu \mathrm{g} / \mathrm{kg}$ and adjusted according to the serum TSH, FT4 and FT3 levels. The diagnosis of $\mathrm{PCH}$ was confirmed due to a high TSH level (38.87 mIU/L) after temporary withdrawal of L-T4 therapy for 4 weeks at the age of 2.3 years. The L-T4 replacement therapy was then resumed. The patient is now 3.1 years of age and receives a daily dose of $6 \mu \mathrm{g} / \mathrm{kg} \mathrm{L}-\mathrm{T} 4$. His physical and intellectual development is appropriate to his age. Family studies showed that his father carried a heterozygous TPO mutation c.2242delT and a heterozygous DUOX2 mutation p. R683L but with no thyroid phenotype, and his mother harboured the heterozygous TPO mutation c.2242delT without abnormal thyroid phenotypes (figure 1).

Patient 2 was diagnosed with $\mathrm{CH}$ in our outpatient paediatric endocrinology clinic. The boy was 4.5 years old at the time; he was $98 \mathrm{~cm}(<3$ th $)$ in height and $13.5 \mathrm{~kg}(<3 \mathrm{th})$ in weight; he had a TSH level of $>100 \mathrm{mIU} / \mathrm{L}$ (normal range $0.7-10 \mathrm{mIU} / \mathrm{L}$ ), and his FT3 and FT4 were $4.2 \mathrm{pmol} / \mathrm{L}$ (normal range 2.65$9.68 \mathrm{pmol} / \mathrm{L}$ ) and $0.3 \mathrm{pmol} / \mathrm{L}$ (normal range 12dence of goitre (right lobe $3.0 \times 1.3 \times 1.2 \mathrm{~cm}$; left lobe $3.2 \times 1.6 \times 1.4 \mathrm{~cm})$. L-T4 replacement therapy was started immediately at an initial daily dose of $15 \mu \mathrm{g} / \mathrm{kg}$ and adjusted according to the serum TSH, FT4 and FT3 levels. The diagnosis of $\mathrm{PCH}$ was confirmed due to a high TSH level $(40.6 \mathrm{mIU} / \mathrm{L})$ after temporary withdrawal of L-T4 therapy for 5 weeks at the age of 6.7 years. At his last visit to our hospital, the patient was 7 years of age and was receiving a daily dose of $6 \mu \mathrm{g} / \mathrm{kg}$ L-T4. His weight was $17.5 \mathrm{~kg}(<3 \mathrm{th})$ and height $113 \mathrm{~cm}$ $(<3$ th). Physical examination and IQ testing showed physical and mental retardation compared to his age. Family studies showed that his father carried a heterozygous TPO mutation p.T561M with no thyroid phenotype (figure 2). Unfortunately, his mother refused to provide a DNA sample. Since biallelic TPO mutations cause goitre and $\mathrm{PCH}$, and the mother was euthyroid with a normal-sized thyroid gland, we speculate that patient 2 had inherited one of the heterozygous variants ( $p$. R648Q/p.T561M) from his mother, and the other $32 \mathrm{pmol} / \mathrm{L})$, respectively. Thyroid ultrasound showed evi-
Figure 4 Structural analysis on p.Thr561Met. Residues around Thr561 at a distance of $10 \AA$ were shown. (A) The wild type of Thr561. (B) The mutation type of Met561. The arrow indicates that the substitution, p.T561M, breaks two hydrogen bonds with an asparagine in position 557 and another hydrogen bond with a glutamic acid in position 558.
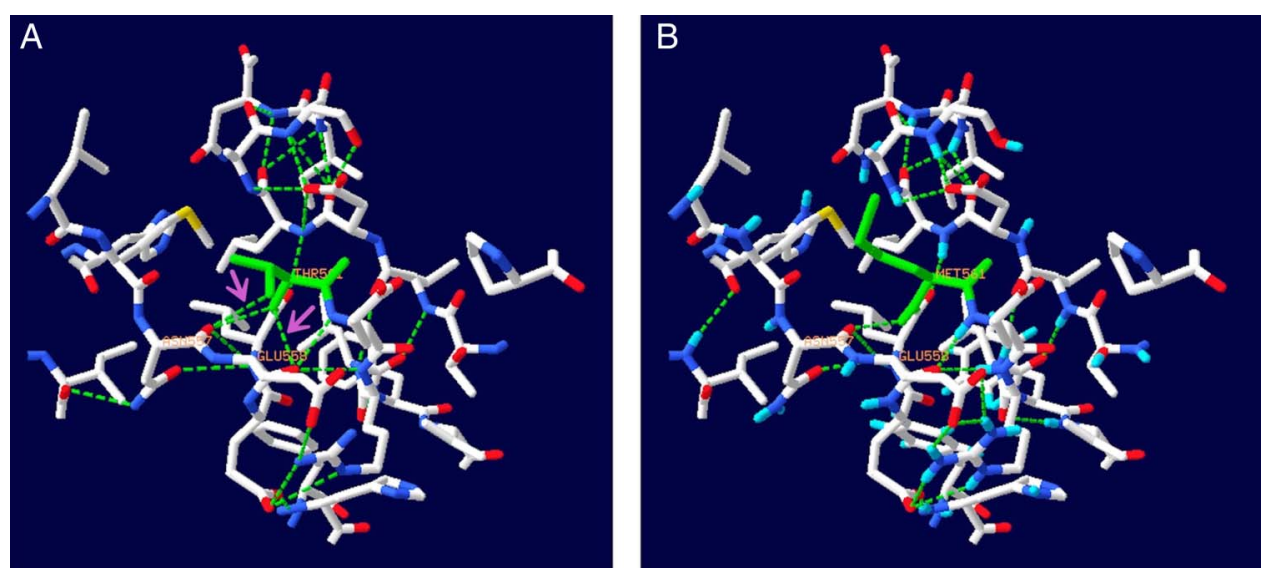


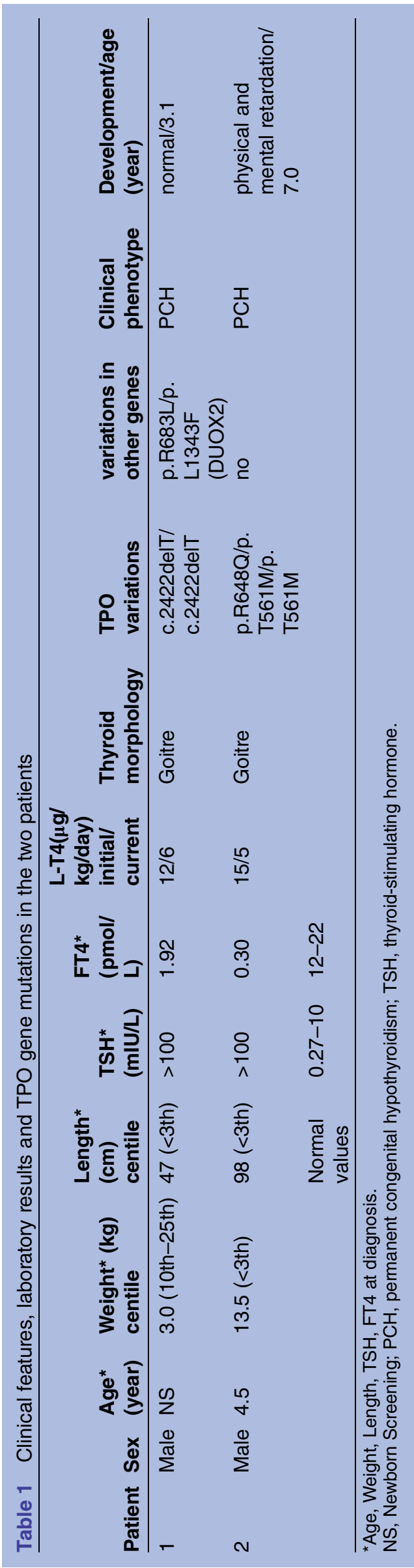

variant was de novo or the patient had inherited both of the variants in the same allele.

\section{DISCUSSION}

In the present study, we conducted one of the largest TPO gene mutation screenings, in a cohort of 192 patients with $\mathrm{CH}$, in the Guangxi Zhuang Autonomous Region, China. The results of our study identified three different TPO variations in two patients with $\mathrm{PCH}$ with enlarged thyroid gland-which was in agreement with the previous notion that defects in TPO cause goitrous $\mathrm{PCH}-$ and revealed a $1 \%$ of TPO mutation rate among patients with $\mathrm{CH}$. The mutation rate is similar to that of Korea $^{18}$ and Iran, ${ }^{19}$ but lower than the rates reported for Portuguese patients with $\mathrm{CH},{ }^{20}$ and Turkish, ${ }^{21}$ Pakistani $^{21}$ and Slovene ${ }^{22}$ patients with TDH. The difference may be explained by the target population and methods involved. Moreover, genetic diversity in each ethnic group may also be implicated.

The present study identified a novel variation: $p$. T561M in the TPO; and four known mutations: c.2422delT and p.R648Q in TPO, and p.R683L and p. L1343F in DUOX2. Cangül $e t a l^{23}$ demonstrated that a homozygous deletion (c.2422delT) in the carboxylterminal coding region of the TPO gene could result in a frameshift mutation and lead to an early stop codon in exon 14 of the gene (p.Cys808AlafsX24), thus causing $\mathrm{CH}$. Pannain et $a l^{24}$ examined three patients with $\mathrm{CH}$ who were heterozygotes for two TPO gene mutations ( $p$. E799K and p.R648Q). Satoh described an 8-year-old boy with normal newborn screening results who developed non-autoimmune hypothyroidism at the age of 1 year and 8 months, and it was found that the boy was heterozygous for R450H-TSHR mutation and double heterozygous for A1323T-DUOX2 and L1343F-DUOX2.$^{25}$ In our recent study, we examined three patients with $\mathrm{CH}$ who had biallelic DUOX2 gene mutations (p.R683L and p. K530X) or triallelic DUOX2 gene mutations (p.R683L/ p.R683L/ p.L1343F and p.R683L/ p.E879K/ p. A1138D). ${ }^{11}$ Structural and in silico functional analysis of the novel candidate missense TPO mutation p.T561M confirmed the causative nature. Threonine in position 561 of the TPO molecule is a highly-conserved amino acid residue. This residue strongly interacts with two other well-conserved residues, asparagine 557 and glutamicacid 558, via hydrogen bonds. Substitution of a neutral polar residue such as threonine by a nonpolar methionine at position 561, destabilises and changes the local structure of protein and is expected to affect amino acid interactions between closer residues of the protein.

Most cases of $\mathrm{CH}$ associated with alterations in the TPO gene are caused by either homozygous or compound heterozygous mutations. In the present study, a mutation homozygous for c.2422delT TPO combined with double heterozygous DUOX2 mutations (p.R683L/ p.L1343F) were identified in patient 1; and triallelic 
TPO pathogenic variants (p.R648Q/p.T561M/p.T561M) were identified in patient 2 . It demonstrated that three or even more variations in one or more $\mathrm{CH}$ associated genes can be found in a single patient, which, in combination, affect the phenotype of the individual. The phenotype of the two patients was severely affected, which was suggested by the extremely low plasma thyroid hormone concentrations, highly elevated TSH levels and high dosage of L-T4. Therefore, the coexistence of multiple mutations may have intensified the severity of the hypothyroid condition.

The genotype-phenotype correlation according to the type of mutation could not be clearly determined yet, because only two patients were identified to be mutation-positive participants and both had multiple variations. Our study demonstrates that the mutational effects from other $\mathrm{CH}$ genes should be taken into account when defining the relationships between mutation genotypes and clinical phenotypes.

In conclusion, we conducted one of the largest TPO mutation screenings, in a cohort of 192 patients with $\mathrm{CH}$, in the Guangxi Zhuang Autonomous Region, China. We identified the prevalence of a TPO pathogenic variant to be $1 \%$ among patients with $\mathrm{CH}$. Three or even more variations in one or more $\mathrm{CH}$ associated gene can be found in a single patient, and the coexistence of multiple mutations may have affected the severity of the hypothyroid condition. A novel TPO variation was reported, which expanded the TPO mutation spectrum and indicated that the TPO mutation rate is very low in the Guangxi Zhuang Autonomous Region, China.

\section{Author affiliations}

${ }^{1}$ Department of Genetic Metabolism, Children's Hospital, Maternal and Child Health Hospital of Guangxi Zhuang Autonomous Region, Nanning, People's Republic of China

${ }^{2}$ Guangxi Center for Birth Defects Research and Prevention, Nanning, People's Republic of China

${ }^{3}$ Endocrinology and Genetic Metabolism of Institute for Pediatric Research, Xinhua Hospital Affiliated to Shanghai Jiaotong University School of Medicine, Shanghai, People's Republic of China

Contributors $\mathrm{CF}$ and $\mathrm{BX}$ participated in the research design, performed the experiments, analysed the data and wrote the paper. SZ, JW, SL, RC, XF and $\mathrm{JL}$ collected clinical samples and analysed the clinical data. BX and $\mathrm{HZ}$ performed the three-dimensional analyses and interpreted the data. $X G$ and $\mathrm{SC}$ proposed the research design, analysed the molecular and overall data, and participated in writing the paper.

Funding This study was supported by the National Natural Science Foundation of China (81260126), Key Projects of Guangxi Health Department (2012025) and Guangxi Natural Science Foundation Programme (2012GXNSFAA053174)

Competing interests None declared.

Patient consent Obtained

Data sharing statement No additional data are available.

Open Access This is an Open Access article distributed in accordance with the Creative Commons Attribution Non Commercial (CC BY-NC 4.0) license, which permits others to distribute, remix, adapt, build upon this work noncommercially, and license their derivative works on different terms, provided the original work is properly cited and the use is non-commercial. See: http:// creativecommons.org/licenses/by-nc/4.0/

\section{REFERENCES}

1. Rastogi MV, LaFranchi SH. Congenital hypothyroidism. Orphanet $J$ Rare Dis 2010;5:17.

2. Kibirige MS, Hutchison S, Owen CJ, et al. Prevalence of maternal dietary iodine insufficiency in the north east of England: implications for the fetus. Arch Dis Child Fetal Neonatal Ed 2004;89:F436-9.

3. Hermanns P, Grasberger H, Cohen R, et al. Two cases of thyroid dysgenesis caused by different novel PAX8 mutations in the DNA-binding region: in vitro studies reveal different pathogenic mechanisms. Thyroid 2013;23:791-6.

4. Park SM, Chatterjee VK. Genetics of congenital hypothyroidism. J Med Genet 2005;42:379-89.

5. Grasberger H, Refetoff S. Genetic causes of congenital hypothyroidism due to dyshormonogenesis. Curr Opin Pediatr 2011;23:421-8.

6. Targovnik HM, Esperante SA, Rivolta CM. Genetics and phenomics of hypothyroidism and goiter due to thyroglobulin mutations. Mol Cell Endocrinol 2010;322:44-55.

7. Belforte FS, Miras MB, Olcese MC, et al. Congenital goitrous hypothyroidism: mutation analysis in the thyroid peroxidase gene. Clin Endocrinol (Oxf) 2012;76:568-76.

8. Ma SG, Qu YL, Zhu H, et al. Novel genetic variants in the TPO gene cause congenital hypothyroidism. Scand J Clin Lab Invest 2015;75:633-7.

9. Bas VN, Aycan Z, Cangul $\mathrm{H}$, et al. A common thyroid peroxidase gene mutation (G319R) in Turkish patients with congenital hypothyroidism could be due to a founder effect. $J$ Pediatr Endocrinol Metab 2014;27:383-7.

10. Ris-Stalpers C, Bikker H. Genetics and phenomics of hypothyroidism and goiter due to TPO mutations. $\mathrm{Mol} \mathrm{Cell}$ Endocrinol 2010;322:38-43.

11. Fu C, Zhang S, Su J, et al. Mutation screening of DUOX2 in Chinese patients with congenital hypothyroidism. J Endocrinol Invest 2015;38:1219-24

12. Cingolani $P$, Platts $A$, Wang le $L$, et al. A program for annotating and predicting the effects of single nucleotide polymorphisms, SnpEff: SNPs in the genome of Drosophila melanogaster strain w1118; iso-2; iso-3. Fly (Austin) 2012;6:80-92.

13. Adzhubei I, Jordan DM, Sunyaev SR. Predicting functional effect of human missense mutations using PolyPhen-2. Curr Protoc Hum Genet 2013:Chapter 7:Unit7 20.

14. Schwarz JM, Rödelsperger C, Schuelke M, et al. MutationTaster evaluates disease-causing potential of sequence alterations. Nat Methods 2010;7:575-6.

15. Arnold K, Bordoli L, Kopp J, et al. The SWISS-MODEL workspace: a web-based environment for protein structure homology modelling Bioinformatics 2006;22:195-201.

16. Schwede T, Kopp J, Guex N, et al. SWISS-MODEL: an automated protein homology-modeling server. Nucleic Acids Res 2003;31:3381-5.

17. Guex N, Peitsch MC. SWISS-MODEL and the Swiss-PdbViewer: an environment for comparative protein modeling. Electrophoresis 1997;18:2714-23

18. Jin HY, Heo SH, Kim YM, et al. High frequency of DUOX2 mutations in transient or permanent congenital hypothyroidism with eutopic thyroid glands. Horm Res Paediatr 2014;82:252-60.

19. Hashemipour M, Soheilipour F, Karimizare S, et al. Thyroid peroxidase gene mutation in patients with congenital hypothyroidism in Isfahan, Iran. Int J Endocrinol 2012;2012:717283.

20. Rodrigues C, Jorge P, Soares JP, et al. Mutation screening of the thyroid peroxidase gene in a cohort of 55 Portuguese patients with congenital hypothyroidism. Eur $J$ Endocrino 2005;152:193-8.

21. Cangul H, Aycan Z, Olivera-Nappa A, et al. Thyroid dyshormonogenesis is mainly caused by TPO mutations in consanguineous community. Clin Endocrinol (Oxf) 2013;79:275-81.

22. Avbelj M, Tahirovic $\mathrm{H}$, Debeljak $\mathrm{M}$, et al. High prevalence of thyroid peroxidase gene mutations in patients with thyroid dyshormonogenesis. Eur J Endocrinol 2007;156:511-19.

23. Cangül H, Doğan $M$, Sağlam $Y$, et al. One base deletion (c.2422delT) in the TPO gene causes severe congenital hypothyroidism. J Clin Res Pediatr Endocrinol 2014;6:169-73.

24. Pannain S, Weiss RE, Jackson CE, et al. Two different mutations in the thyroid peroxidase gene of a large inbred Amish kindred: power and limits of homozygosity mapping. J Clin Endocrinol Metab 1999;84:1061-71.

25. Satoh M, Aso K, Ogikubo S, et al. Hypothyroidism caused by the combination of two heterozygous mutations: one in the TSH receptor gene the other in the DUOX2 gene. J Pediatr Endocrinol Metab 2015;28:657-61. 